\title{
Semantic Standards Quality Measured for Achieving Enterprise Interoperability: The Case of the SETU Standard for Flexible Staffing
}

\author{
Erwin Folmer * - Harris Wu ** \\ *University of Twente \& TNO, The Netherlands \\ erwin.folmer@tno.nl \\ ** Old Dominion University, USA \\ hwu@odu.edu
}

\begin{abstract}
Semantic standards should play an important role in achieving inter-organizational interoperability. Millions are spent on development and adoption of these standards, but does it lead to interoperability? This important question is often not addressed. In this study data interoperability in the Dutch temporary staffing industry is studied by focusing on the quality of the SETU standard and its implementations in practice. The Stichting Elektronische Transacties Uitzendbranche (foundation for electronic transactions in the staffing industry,) or SETU, develops and maintains standards for exchange of electronic data in the staffing industry. Our results show that although the SETU standard is equipped for achieving interoperability, this in practice has not been achieved due to low quality implementations. We raise the question why these studies are not being performed on every standard. Another result is that localizations (profiles) may be needed for high quality standards; without localizations interoperability is limited in the SETU case.
\end{abstract}

KEYWORDS: semantic standards, standardization, interoperability, quality 


\section{Introduction}

Achieving interoperability in many industries is challenging but has great impact. Studies of the US automobile sector, for example, estimate that insufficient interoperability in the supply chain adds at least one billion dollars to operating costs, of which $86 \%$ is attributable to data exchange problems (Brunnermeier and Martin 2002). Later studies mention5 billion dollars for the US automotive industry and 3.9 billion dollars for the electro technical industry, both representing an impressive $1.2 \%$ of the value of shipments in each industry (Steinfield et al. 2011). The adoption of standards to improve interoperability in the automotive, aerospace, shipbuilding and other sectors could save billions (Gallaher et al. 2002).

The already huge importance of standards and interoperability will continue to grow. Networked business models are becoming indisputable reality in today's economy (Legner and Lebreton 2007). A recent Capgemini study concludes that to be ready for 2020 companies need to "significantly increase their degree of collaboration as well as their networking capability" (Falge et al. 2012).

Standards are important for ensuring interoperability (Rada 1993). "Standards are necessary both for integration and for interoperability" (Dogac et al. 2008). "Adopting standards-based integration solutions is the most promising way to reduce the long-term costs of integration and facilitate a flexible infrastructure" (Chari and Seshadri 2004). Some go even further: "Inter-organizational collaboration requires systems interoperability which is not possible in the absence of common standards" (Gerst et al. 2005). And the potential of standards, in relation to the problematic introduction of proprietary solutions, is shown in a case study from the automotive industry (Steinfield et al. 2011).

There is hardly any research on the achievements of semantic standards in achieving interoperability. A survey among semantic standards organizations shows that the vast majority believe that their standards can be improved, and that improvements will lead to more interoperable systems. However, standard developers need statistical support to find the needed improvements (Folmer et al. 2011).

\section{Background}

\subsection{Semantic Standards Defined}

Semantic standards reside at the presentation and application layer of the OSI model (Steinfield et al. 2007). They include business transaction standards, interorganizational information system (IOS) standards, ontologies, vocabularies, 
messaging standards, document-based, e-business, horizontal (cross-industry) and vertical industry standards. The often used examples are RosettaNet for the electro technical industry, HealthLevel7 for the health care domain, HR-XML for the human resources industry and Universal Business Language (UBL) for procurement. Semantic standards are designed to promote communication and coordination among organizations; these standards may address product identification, data definitions, business document layout, and/or business process sequences (adapted from (Steinfield et al. 2007)).

\subsection{Quality Measured in Application: Relevance and Completeness}

Zhu \& Wu have introduced how relevance and completeness can be measured in standards' implementations (Zhu and $\mathrm{Fu}$ 2009; Zhu and $\mathrm{Wu} 2010$; Zhu and $\mathrm{Wu}$ 2011). The completeness and relevancy of the same data standard can be different to different users. Further, they can be different between an individual user and the user community. To formalize the metrics, let the $\mathbf{S}$ be the set of data elements specified in the data standard, $\mathbf{U}_{\mathbf{i}}$ be the data elements required by the user i. From the user i's perspective, the metrics can be defined as (Zhu \& Wu 2010)

$$
\text { Completeness }_{i}=\frac{\left|U_{i} \cap S\right|}{\left|U_{i}\right|} \text {, and Relevancy } \text { Re }_{i}=\frac{\left|U_{i} \cap S\right|}{|S|}
$$

From the user community's perspective, the metrics can be defined as

$$
\text { Completeness }_{c}=\frac{\left|\left(\bigcup_{i} U_{i}\right)\right| S \mid}{\left|\bigcup_{i} U_{i}\right|} \text {, and Relevancy } c=\frac{\left|\left(\bigcup_{i} U_{i}\right)\right| S \mid}{|S|}
$$

Although the concept of completeness and relevancy is a limited, incomplete view on standard quality, it is an important contribution since it is the only notion of quality in practice that can be objectively measured using a large number of data instances.

$\mathrm{Zhu}$ and $\mathrm{Wu}$ focused on the standard of public financial reporting in US based on US-GAAP (United States Generally Accepted Accounting Principles). This reporting standard is one of the most important standards based on XBRL (Extensible Business Reporting Language). Thousands of companies are mandated to report quarterly and annual financial reports to US Stock and Exchange Commission, using the US-GAAP XBRL standard. Applying automated tools to thousands of public financial reports, $\mathrm{Zhu}$ and $\mathrm{Wu}$ were able to perform a series of analyses on the quali- 
ty of US-GAAP XBRL standard and interoperability of public financial reports, including trend and industry-based analyses. Most importantly, Zhu and Wu's studies produced a number of practical suggestions to both the standard defining body and standard users/implementers (i.e. the individual reporting companies), which may substantially improve standard compliance and data interoperability.

The XBRL standard in Zhu and Wu's studies are used in financial reports, which are relatively static information. No researchers have yet studied the quality of electronic standards used for dynamic business processes, using similar implementationbased metrics.

\section{The SETU Standard}

SETU stands for the Stichting Elektronische Transacties Uitzendbranche (foundation for electronic transactions in the staffing industry). The SETU standard is a semantic standard trying to achieve interoperability among different actors in the business processes related to flexible staffing. The staffing industry consists of a large number of customers and suppliers, and increasingly relies on electronic transactions.

The SETU standard has been acclaimed by the Dutch government for achieving interoperability within the process of hiring temporary staffers through staffing organizations. Since May 2009, SETU is listed on the "comply or explain" list, which means that every (semi) public organization in the Netherlands has to comply with using the SETU standard when ordering temporary staffing is executed electronically. Achieving this status implies that SETU passed the process of the Dutch government, suggesting that SETU is expected to be of high quality.

SETU is a set of specifications, including XML Schemas, for amongst others assignments, timecards, and invoices related to temporary staffing. It is a Dutch localization of the international HR-XML standard (Van Hillegersberg \& Minnecre, 2009). SETU standardizes additional rules on top of HR-XML, and thereby limits the options within HR-XML. An instance that validates correctly with HR-XML does not necessarily comply with SETU. However the other way around is always true: Each instance that is SETU compliant is compliant to HR-XML as well.

Since SETU uses the same XML Schema files as HR-XML, the additional rules are captured within text (the SETU specifications) and the business rules in Schematron. The SETU standard comprises of different sub-standards. Our focus is on SETU standard for invoicing version 1.1, which is freely available at www.setu.nl. 


\section{Research Approach}

On a high level we want to know if we can assess the quality of the standard by assessing the implementations of the standard. We study this question by performing a case study on the SETU standard. We selected this standard because it is important to workforce mobility and integration, and is highly acclaimed for its quality, adoption success and business savings. Our main research question is: What is the quality of the SETU standard and its implementations?

To be able to answer this question we have to analyze the implementations of the SETU standard and search for (avoidable) errors that negatively affect the quality of both standard and implementations. The SETU standard has to be compared with other standards in terms of quality but so far only quality results from XBRL are available for comparison.

Our second research aim is to study whether this implementation measuring approach is useful in identifying improvement suggestions for semantic standards. In this specific case we will identify improvement suggestions for the SETU standard. In other words, can our approach be used as quality assessment instruments requested by standard developers (Folmer et al. 2011)?

\subsection{Data Collection}

For our research approach we need to collect data of SETU implementations. One of the authors contacted the main staffing organizations and system providers that together have the vast majority in market share of temporary staffing in the Netherlands.

Data of four large staffing organizations has been gathered, just as the data from 3 system providers, which have been anonymized. In total 54 "messages" have been gathered including 32 timecards and 22 invoices. These messages are instantiations based on the SETU standards: For instance a specific invoice for worker X, week Y, sent by staffing organization A to staffing customer B. The data then contains invoices from staffing organizations to staffing customers.

There is some overlap in data, since the staffing customer may overlap for different instantiations of staffing organizations. Also, some staffing organizations make extensively use of system providers. Therefore the data gathered from the system providers may include the same data as received from a staffing organization. 


\subsection{Validation Process}

As first step we decided to take one set of message: the invoices. From our data set 22 are invoices, of which 2 have the same system-staffing organization - staffing customer configuration and will therefore have the same characteristics.

These two have been removed from the data set. Our approach consisted then of 3 steps:

- Validate the messages in the SETU eValidator. This Validation Service is available at www.setu.nl and validates the instances three way: a. XML wellformedness, $b$. XML Schema validation and c. Business Rules validation. The latter is a set of Schematron expressions that has been set up by the SETU organization that encapsulates additional rules described in the SETU specification that cannot be validated with XML Schema.

- Count the usage of elements within the implementations; by doing that we find frequently used or totally unused elements.

- Analyze and calculate metrics. Within this step we analyze the previous results and calculate error percentages and the completeness and relevance metrics.

\section{SETU Measurement Results for the Invoice standard}

This section contains the results of the measurement of the SETU invoice implementations. We will start by explaining the validation results, followed by looking at more details into the data elements. Finally we will discuss the results on relevance and completeness metrics.

\subsection{Validation Errors}

Table 1 contains the validation results. For each usage scenario (such as StaffinOrg1 invoicing StaffingCustomer1), the numbers of XML structure errors, HR-XML schema validation errors and SETU business rules errors are counted. Although most errors are counted by occurrences, when structure errors occur the tool may exit immediately and therefore a yes/no is used for scoring. 


\begin{tabular}{|l|l|l|l|l|l|}
\hline$\#$ & Usage scenario (invoice type) & $\begin{array}{l}\text { Structure } \\
\text { Errors }\end{array}$ & $\begin{array}{l}\text { Schema } \\
\text { Errors }\end{array}$ & $\begin{array}{l}\text { Rule } \\
\text { Errors }\end{array}$ & $\begin{array}{l}\text { Total } \\
\text { Errors }\end{array}$ \\
\hline 1 & StafOrg1-StafCust1 & No & 0 & 2 & 2 \\
\hline 2 & StafOrg1-StafCust2 & No & 0 & 2 & 2 \\
\hline 3 & StafOrg1-StafCust3 & No & 0 & 2 & 2 \\
\hline 4 & StafOrg1-StafCust4 & No & 0 & 2 & 2 \\
\hline 5 & StafOrg1-StafCust5 & No & 0 & 2 & 2 \\
\hline 6 & StafOrg1-StafCust6 & No & 0 & 2 & 2 \\
\hline 7 & StafOrg2-StafCust1 & No & 0 & 3 & 3 \\
\hline 8 & StafOrg2-StafCust2 & No & 0 & 1 & 1 \\
\hline 9 & StafOrg2-StafCust3 & No & 0 & 3 & 3 \\
\hline 10 & StafOrg2-StafCust4 & No & 0 & 3 & 3 \\
\hline 11 & StafOrg3-StafCust3 & No & 1 & 2 & 3 \\
\hline 12 & StafOrg3-StafCust4 & No & 11 & 14 & 25 \\
\hline 13 & StafOrg4-StafCust1 & No & 0 & 4 & 4 \\
\hline 14 & StafOrg4-StafCust2 & No & 0 & 4 & 4 \\
\hline 15 & StafOrg4-StafCust3 & No & 16 & 4 & 20 \\
\hline 16 & System1-StafOrg1-StafCust1 & No & 0 & 2 & 2 \\
\hline 17 & System1-StafOrg1-StafCust2 & No & 0 & 2 & 2 \\
\hline 18 & System1-StafOrg2-StafCust3 & No & 0 & 2 & 2 \\
\hline 19 & System1-StafOrg3-StafCust4 & No & 1 & $-*$ & $1 *$ \\
\hline 20 & System3-StafOrg1-StafCust1 & Yes & 7 & 3 & 10 \\
\hline & Invoice Errors & & 36 & 59 & 95 \\
\hline
\end{tabular}

Table 1. Validation results for SETU invoices

\subsection{Validation Data Explanation}

The * denotes the fact that due to the schema errors the business rules validation was cancelled. We received more invoices of the system implementations, but since they have the same errors we excluded them from the data set. This is logical since when the invoice standard is implemented in the system of the staffing organizations, even if we collect 1,10 or 1000 instances of the invoice, they will all have the same errors. System 2 is excluded from this set because it has only timecards implemented and no invoices.

The implementations gathered from an organization often contain the same characteristics and errors. For example Staffing Organization 1 succeeded in their implementation to avoid schema errors. Some staffing organizations have their own ICT implementation, while others use a system from a software vendor. 
StaffingOrg 4 has 16 schema errors which are in fact the same error that returns in many places. It shows that the number of errors in itself is not always useful. For instance if the amount is missing on an invoice line, and there are 20 invoice Lines, than it will count as 20 rule errors.

These explanations show that the analysis is valuable; however, carefulness is needed when quantifying measurements.

\subsection{Validation Data Analysis}

It is valuable to summarize the numbers of implementations that have or have not errors. Below are some statistics:

- 1 out of 20 is not well-formed, and has basic structure errors

- 5 out of 20 have basic XML Schema error and do not validate against schema

- 19 out of 19 (100\%) have Business Rules error and do not comply to the SETU standard

The first two statistics can be easily manipulated by changing the data set, but still it is remarkable that these errors exist in practice. The fact that $100 \%$ contain business rules errors and no instance is SETU compliant is even more remarkable. If we look in detail into the errors:

- XML Schema errors:

A wide range of "clumsy" errors. For instance incorrect use of date notation within DocumentDateTime element, a missing element, or usage of a non-existent value from the code list. All these errors can be easily avoidable.

- Business Rules:

In contrast to the XML Schema errors, the Business Rules errors have a lot of similarities in all implementations. We found two groups of errors:

Group 1: The usage of "SupplierParty", "BillToParty" and "RemitToParty" leads to many errors.

Group 2: The usage of "Invoice Lines" without "Amount" or "Price per Quantity".

It seems like implementers have difficulties with using these elements correctly. This might indicate that the specification is not clear about how to use these elements. 


\subsection{Data Elements}

Our analysis shows that several data elements from the standard are never used within our data set. These unused elements are: DiscountAmount, DiscountPercentage, DepartmentCode, and CostCenterName. On the other hand there are several data elements that are used in every instance: DocumentDateTime, Header, Id, IdValue, Invoice, Line, LineNumber, PercentQuantity, TaxBaseAmount, Total, TotalAmount, TotalTax, Type. The customer reporting requirement module is a set of optional elements that allow flexible usage for specific needs of staffing customers. The most used items of the customer reporting requirements are listed in table 2.

\begin{tabular}{|l|l|}
\hline Element & Count \\
\hline PurchaseOrderLineItem & 14 \\
\hline AdditionalRequirement & 12 \\
\hline CostCenterCode & 10 \\
\hline PurchaseOrderNumber & 8 \\
\hline CustomerReferenceNumber & 6 \\
\hline CostCenterName & 2 \\
\hline ProjectCode & 1 \\
\hline
\end{tabular}

Table 2. Most used items from Customer Reporting Requirements

The list of frequencies of these optional elements provides many insights. For instance since PurchaseOrderLineItem is used that often, it might be questioned whether the element should be mandatory. Additional to the optional elements within the Customer Reporting Requirements, the SETU standard allows users to define custom fields in the "AdditionalRequirement" element. Out the 22 invoices, 16 of them have defined AdditionalRequirement with the list of custom fields below. Several organizations independently developed same or similarly-titled custom fields, which may suggest a need for these elements to be included in the SETU standard.

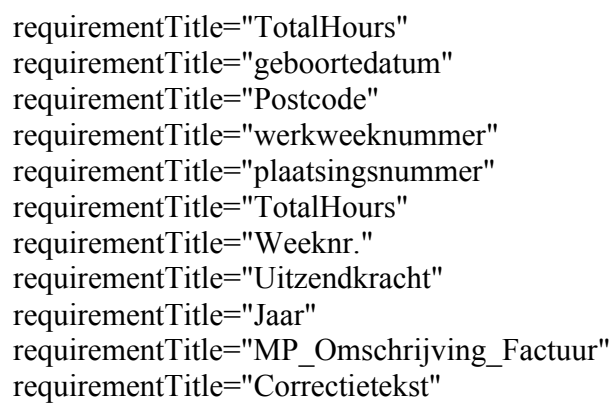




\subsection{Completeness and Relevance}

HR-XML standard contains 385 elements. SETU invoicing standard defined 78 elements, 55 of which are from the HR-XML standard. Considering SETU as the user of the HR-XML standard, the relevancy of HR-XML is $14.29 \%$, while the completeness is $70.51 \%$.

\begin{tabular}{|l|l|l|l|}
\hline Standard User & Standard & Completeness & Relevancy \\
\hline SETU & HR-XML & $55 / 78=70.51 \%$ & $55 / 385=14.29 \%$ \\
\hline SETU community & $\begin{array}{l}\text { SETU } \\
\text { Invoicing } \\
\text { Standard }\end{array}$ & 1 & $74 / 78=94.87 \%$ \\
\hline $\begin{array}{l}\text { SETU community } \\
\text { considering } \\
\begin{array}{l}\text { AdditionalRequirement as } \\
\text { custom elements }\end{array}\end{array}$ & $\begin{array}{l}\text { SETU } \\
\text { Invoicing } \\
\text { Standard }\end{array}$ & $74 / 85=87.06 \%$ & $74 / 78=94.87 \%$ \\
\hline
\end{tabular}

Table 3. SETU results on completeness and relevance

All invoices in our data collection are specified using elements specified in SETU. For SETU community, most of the 78 SETU elements have been used in invoices. The relevancy of SETU invoicing standard is $94.87 \%$. All elements used in invoices are defined in SETU invoicing standard, therefore its completeness is 1. However, if the custom fields in AdditionalRequirement are considered as custom elements, the completeness of SETU standard in the context of our data collection is $87.06 \%$.

\section{Discussion}

Most remarkable is the outcome that no single instance is a correct implementation of the standard. The SETU workgroup should analysis these results. But based on these results we suggest:

-To clarify the use of SupplierParty, RemitToParty and BillToParty

-To clarify the use of Invoice Lines that requires amounts and price per quantity.

-To remove the unused elements: this makes implementations easier

-To change the cardinality to mandatory of the always used elements: again, this makes implementations easier

-Analyze the results of the usage of the customer reporting module 
Remarkable is that we used the eValidator in our study, which is accessible through the SETU.NL website. Our results make it questionable if this validation tool is currently used by the SETU implementers. We guess that the quality of the implementations would have been better if the implementers had used the validation service freely available to them.

\subsection{Comparison of SETU and XBRL Results}

Zhu and Wu (2011)'s study finds that for the US-GAAP XBRL user community, the completeness of US-GAAP standard is $32.12 \%$ and the relevancy is $19.29 \%$. Their study is based on all annual financial reports that have been submitted to US Stock and Exchange Commission as of 2010. SETU invoicing standard seems to have a better fitness for use by the staffing community. As measured by our data collection, SETU invoicing standard's completeness is $87.06 \%$, and relevancy is $94.87 \%$. However, note that if the data collection is larger, such as if we were able to collect all invoices from the staffing community in Netherlands, the completeness of SETU invoicing standard would have been somewhat lower (considering custom fields in AdditionalRequirement as custom elements). Also, financial reports are much more complex than timesheets and invoices. The US-GAAP standard has more than 12000 elements and is much more complex than the SETU invoicing standard. The lower fitness for use of US-GAAP XBRL standard, as measured by completeness and relevancy, can be partly attributed to its complexity.

The results show the value of having localizations on top of broader semantic standards (such as HR-XML), in line with earlier findings that localizations are essential for interoperability (Brutti et al. 2011).

\subsection{Discussion on Potential Reasons of Outcome}

The results are highly remarkable and definitely not expected for SETU. Therefore we discussed the outcome with both developers of the SETU standard, and its users. Based on these discussions we defined the following potential reasons for the low quality of the implementations:

1. Early stage of SETU standard in life cycle. The SETU standard is relatively new, and implementations have mainly started from 2009 onwards. So the tested implementations are probably from the first batch of implementations. It might be expected that during the years both the standards and its implementations have been improved. Follow-up research in which this validation is repeated is required to test this assumption. 
2. Plug-and-play interoperability, the goal of standards such as SETU, is not desired on business level. This might be surprising since plug-and-play interoperability is seen as some kind of holy grail since the uprise of e-business, and in particular the ebXML years. However in discussion with business people from the staffing companies they state that $90 \%$ interoperability is better than $100 \%$. Simply because they would like to have contact with the customer about the final $10 \%$. Plug and play would reduce that customer contact and will reduce the feeling the customer will have with the staffing company. So far academia is convinced that $100 \%$ plug and play should be the goal, but this research might question that. Validation of the suspected $100 \%$ plug and play requirement from business perspective is needed.

3. SETU is used as a marketing term. Nobody cares about the correctness of the implementations. For especially the staffing companies and system providers it is essential to state that they are SETU compliant. Even during the early years most companies said they had implemented SETU although these statements were highly questionable. Customers are asking for SETU, especially in large government tenders, and failing to state SETU compliancy might cost contracts. However since implementations are never tested, and there is no official SETU compliance organized, it might lead to issues regarding implementations as shown in this study.

4. Knowledge problem of implementers, both on semantics (business) level and technical level. On semantic level, it is hard to understand the real meaning of the semantics if the implementer did not participate in the SETU workgroup. However our study is not focused on semantic errors, but on technical errors. There might even be more errors on semantic level, but these are hard to find. Second there might be a mismatch between the technology used within the SETU standard and the technical knowledge of the implementers. Although this is hard to imagine since SETU is using very traditional XML technology. On the other side the IT maturity of the temporary staffing industry is expected to be low.

For this, and the previous reason, certification might be a potential solution to improve the implementations.

5. The flexibility of the standard, especially during early stages of life cycle. There is always a debate on how flexible a standard should be and how many changes and version updates are needed to accommodate the community. This is not much likely to be a real reason since not many of the errors are related to issues that later on have been accommodated within the standard.

6. Standard is being used in situations that are not foreseen or explicitly mentioned as use case. Within the SETU case the standard is explicitly designed for a situation in which staffing customer and staffing company directly exchange the messages. However in practice the standard is also being used in situations with an 
intermediary system provider in the middle. The requirements of that situation might not be taken into account during the SETU standards development and might lead to implementation issues.

Although we searched and discussed the reasons in particular related to the SETU implementations, these reasons might as well be valid for other semantic standards.

\section{Conclusions}

This paper has shown an analysis of the implementations of a semantic standard. The quality of SETU implementations is highly questionable since no single instance proved to be a correct implementation. The quality of SETU standard seems to be ok compared to other electronic standards such as the US-GAAP XBRL standard in financial reporting.

We have provided valuable results to the SETU standards organization, in two ways:

1. The low quality of implementations raises the question what the SETU organization can do to improve the implementations. Education, mandatory validation, or even certification might be solutions.

2. This analysis has been used for an improvement project within SETU. The analysis of element usage frequency and the two groups of frequent errors might lead to changes in new version of the standard.

We have provided supporting evidence to earlier claims that localizations (profiles) are needed for achieving interoperability. But our study also shows that it is not enough to have a single view on standard quality, especially when studying the interoperability effect of standards. SETU scored almost perfectly on completeness and relevance, but still interoperability was questionable due to low quality of implementations. The other way around could be said for other standards. At minimum an interoperability achievement study should include a study of:

- Completeness and Relevance of the standard

- Validity of standard implementations

It is remarkable that no semantic standardization organizations are using above measures to improve their standards, or other quality measurement approaches (Folmer, 2012). Based on our research we strongly advise all semantic standardization workgroups to perform such an analysis and improve the standards or set up policies to improve implementations. 


\section{References}

Brunnermeier, S.B., and Martin, S.A. "Interoperability Costs in the Us Automotive Supply Chain," Supply Chain Management, vol. 7 no. 2, 2002, p. 71-82.

Brutti, A., De Sabbata, P., Frascella, A., Novelli, C., and Gessa, N. "Standard for Ebusiness in Smes Networks: The Increasing Role of Customization Rules and Conformance Testing Tools to Achieve Interoperability," in Enterprise Interoperability, Proceedings of the Workshops of the Third International Ifip Working Conference Iwei 2011, M. Zelm, M. Van Sinderen, G. Doumeingts and P. Johnson (eds.). Stockholm: Wiley.

Chari, K., and Seshadri, S. "Demystifying Integration," Communications of the ACM, vol 47 no 7,2004, p. 58-63.

Dogac, A., Kabak, Y., Namli, T., and Okcan, A. 2008. "Collaborative Business Process Support in Ehealth: Integrating Ihe Profiles through Ebxml Business Process Specification Language," IEEE Transactions on Information Technology in Biomedicine ,vol 12 no 6,2008, p. 754-762.

Falge, C., Otto, B., and Österle, H. "Data Quality Requirements of Collaborative Business Processes," 45th Hawaii International Conference on System Sciences (HICSS),2012, Hawaii.

Folmer, E., Oude Luttighuis, P., and van Hillegersberg, J. "Do Semantic Standards Lack Quality? A Survey among 34 Semantic Standards," Electronic Markets ,vol 21 no 2, 2011, p. 99-111.

Gallaher, M.P., O'Conner, A.C., and Phelps, T. "Economic Impact Assessment of the International Standard for the Exchange of Product Model Data (Step) in Transportation Equipment Industries," 2002, RTI Project Number 07007.016.

Gerst, M., Bunduchi, R., and Williams, R. "Social Shaping \& Standardization: A Case Study from Auto Industry," 38th Hawaii International Conference on System Sciences (HICSS), J.R.H. Spraque (ed.), 2005, Hawaii.

Legner, C., and Lebreton, B. 2007. "Preface to the Focus Theme Section: 'Business Interoperability' Business Interoperability Research: Present Achievements and Upcoming Challenges," Electronic Markets ,vol 17 no 3 ,2007, p. 176-186.

Rada, R. 1993. "Standards: The Language for Success," Communications of the ACM ,vol 36 no 12,1993 , p. $17-23$.

Steinfield, C.W., Markus, M.L., and Wigand, R.T. "Cooperative Advantage and Vertical Information System Standards: An Automotive Supply Chain Case Study," 44th Hawaii International Conference on System Sciences (HICSS), 2011, Hawaii.

Steinfield, C.W., Wigand, R.T., Markus, M.L., and Minton, G. "Promoting E-Business through Vertical Is Standards: Lessons from the Us Home Mortgage Industry," in Standards and Public Policy, S. Greenstein and V. Stango (eds.). Cambridge: Cambridge University Press, 2007, p. 160-207.

Zhu, H., and Fu, L. "Towards Quality of Data Standards: Empirical Findings from Xbrl," International Conference on Information Systems (ICIS), 2009, Phoenix. 
Zhu, H., and Wu, H. "Quality of Data Standards: Framework and Illustration Using Xbrl Taxonomy and Instances," Electronic Markets ,vol 21 no 2,2011 , p. 129-139.

Zhu, H., and Wu, H. "Quality of Xbrl Us Gaap Taxonomy: Empirical Evaluation Using Sec Filings," Americas Conference on Information Systems (AMCIS), 2010, Lima, Peru. 